PROCEEDINGS OF THE

AMERICAN MATHEMATICAL SOCIETY

Volume 127, Number 4, April 1999, Pages 1221-1230

S 0002-9939(99)04628-6

\title{
ON SUBSPACES OF PSEUDORADIAL SPACES
}

\author{
ALAN DOW AND JINYUAN ZHOU \\ (Communicated by Carl Jockusch)
}

\begin{abstract}
A topological space $X$ is pseudoradial if each of its non closed subsets $A$ has a sequence (not necessarily with countable length) convergent to outside of $A$. We prove the following results concerning pseudoradial spaces and the spaces $\omega \cup\{p\}$, where $p$ is an ultrafilter on $\omega$ :

(i) $\mathrm{CH}$ implies that, for every ultrafilter $p$ on $\omega, \omega \cup\{p\}$ is a subspace of some regular pseudoradial space.

(ii) There is a model in which, for each P-point $p, \omega \cup\{p\}$ cannot be embedded in a regular pseudoradial space while there is a point $q$ such that $\omega \cup\{q\}$ is a subspace of a zero-dimensional Hausdorff pseudoradial space.
\end{abstract}

\section{INTRODUCTION AND DEFINITIONS}

In [1] the authors asked if $\omega \cup\{p\}$ is a subspace of a pseudoradial space for $p \in \beta \omega \backslash \omega$, where $\omega \cup\{p\}$ takes the subspace topology in $\beta \omega$, i.e, $p$ is the only nonisolated point and a neighbourhood of $p$ is $A \cup\{p\}$ for $A \in p$. It is proved in [9] that under the assumption $\mathfrak{p}=\mathfrak{c}$, each space with countable tightness is a subspace of some pseudoradial space. It is essentially proved that, under the assumption $\mathfrak{p}=\mathfrak{c}$, $\omega \cup\{p\}$ is a subspace of a Hausdorff pseudoradial space for $p \in \beta \omega \backslash \omega$. However the pseudoradial space constructed in [9] is not regular. In a communication with the second author, J. Vaughan asked if it is possible to make the pseudoradial space in [9] regular. In [7] P. Nyikos asked whether each topological space can be embedded into a pseudoradial space (Problem 6.22). Our discussion will be focused on the class of regular spaces. We first prove, in section 2 , that, under $\mathrm{CH}, \omega \cup\{p\}$ is a subspace of a regular pseudoradial space for each ultrafilter $p$ on $\omega$. However, the question of whether $\mathrm{CH}$ can be replaced by MA remains open. In section 3, we prove that if the ground model satisfies $\mathrm{CH}$, then in the forcing extension obtained by adding $\aleph_{2}$ many Cohen reals, $\omega \cup\{p\}$, for any P-point $p$, is not a subspace of any regular pseudoradial space. In section 4 , we show that, for a special ultrafilter $p$ constructed in [5], if there exists a special stationary set and $\mathfrak{c}=\omega_{2}$, then $\omega \cup\{p\}$ can be embedded into a zero-dimensional Hausdorff pseudoradial space. We also prove that it is consistent that $\mathfrak{c}=\omega_{2}$ while there is such a stationary set. We conclude that there is a forcing extension in which, for every P-point $p, \omega \cup\{p\}$ cannot be embedded in any regular pseudoradial space while there is an ultrafilter $q$ on $\omega$ such that $\omega \cup\{q\}$ is a subspace of a zero-dimensional Hausdorff pseudoradial space.

Received by the editors March 17, 1997 and, in revised form, July 30, 1997.

1991 Mathematics Subject Classification. Primary 54E35.

Key words and phrases. Forcing, $\mathrm{CH}$, ultrafilter, zero-dimensional space, pseudoradial.

(C)1999 American Mathematical Society 
Definition 1.1. A sequence $\left\{x_{\alpha}: \alpha<\lambda\right\}$ of points in $X$ is said to be convergent to $x$ provided that for each neighbourhood $U$ of $x$, there exists $\alpha<\lambda$ such that $\left\{x_{\beta}: \beta>\alpha\right\} \subseteq U$. (We will call any transfinite sequence a sequence.)

A space $X$ is radial if for each subset $A$ of $X$ and $x$ in $\bar{A}$, there is a sequence in $A$ convergent to $x$. The pseudoradial spaces have weaker properties. This is analogous to the relationship between sequential spaces and Frechét spaces.

Definition 1.2. A subset $A$ of a space of $X$ is said to be radially closed if no sequence in $A$ is convergent to outside of $A$. A space $X$ is said to be pseudoradial provided that any radially closed subset of $X$ is closed in $X$.

In this paper, a topological space is always regular. The set theory notions are standard. For example, if $A$ and $B$ are two sets, we write $A={ }^{*} B$ when $A$ and $B$ are equal modulo finite.

\section{UNDER CH}

If $p$ is a $\mathrm{P}_{\mathfrak{c}}$ point on $\omega$, let $\left\{A_{\alpha}: \omega \leq \alpha<\mathfrak{c}\right\}$ be a strictly decreasing modulo finite base for $p$ and let $B$ be the Boolean Algebra generated by $\left\{A_{\alpha}: \alpha \in \mathfrak{c}\right\} \cup[\omega]^{<\omega}$; then it is easy to see that the Stone space of $B$ is just $\omega \cup[\omega, \mathfrak{c}+1]$ where $[\omega, \mathfrak{c}+1]$ has the usual ordinal topology and $\omega \cup\{\mathfrak{c}\}$ is homeomorphic to $\omega \cup\{p\}$. An immediate corollary is that, under $\mathrm{CH}$, if $p$ is a $P$-point, then $\omega \cup\{p\}$ can be embedded into a zero-dimensional Hausdorff compact pseudoradial space.

However, we have the following result about non $P$-points.

Lemma $2.1(\mathrm{p}=\mathfrak{c})$. If $p$ is not a P-point, then $\omega \cup\{p\}$ can be embedded in a zerodimensional pseudo-radial space.

Proof. Let $p=\left\{A_{\alpha}: \alpha<\mathfrak{c}\right\}$ such that $\left\{A_{i}: i<\omega\right\}$ witnesses that $p$ is not a $P$-point. First of all, we will construct a base $\left\{B_{\alpha}: \alpha<\mathfrak{c}\right\}$ of $p$ and an almost disjoint family $\left\{C_{\alpha}: \alpha<\mathfrak{c}\right\}$ such that, for $\xi, \alpha \in \mathfrak{c}$, the following are true:

(i) $)_{\alpha} \quad B_{\alpha} \subseteq A_{\alpha}$,

(ii) $\xi_{\alpha} \quad \xi \leq \alpha \rightarrow C_{\alpha} \subseteq^{*} B_{\xi}$,

(iii) $\xi \alpha \quad \alpha<\xi \rightarrow C_{\alpha} \cap B_{\xi}={ }^{*} \emptyset$.

Without loss of generality, we can assume that $\left\{A_{n}: n \in \omega\right\}$ is strictly decreasing with empty intersection. We also assume that $A_{0}=\omega$. It is easy to construct $\left\{B_{\xi}, C_{\xi}: \xi \leq \omega\right\}$ with $B_{n}=A_{n}$ for $n \in \omega$. Suppose we have constructed $\left\{B_{\xi}, C_{\xi}\right.$ : $\xi<\alpha\}$ with $\alpha \geq \omega$ and such that $(i)_{\xi},(i i)_{\xi \eta}$ and $(i i i)_{\xi \eta}$ are true for each $\xi, \eta<\alpha$. We have to define $B_{\alpha}$ and $C_{\alpha}$. For $\xi \in \alpha$ and $n \in \omega$, if $\xi \geq \omega$, then $C_{\xi} \subseteq^{*} A_{n}$. By the fact that $\mathfrak{p}=\mathfrak{c}$, there is a $D \subseteq \omega$ such that $D$ is a pseudo-intersection of $\left\{A_{n}: n \in \omega\right\}$ and, for $\omega \leq \xi<\alpha, C_{\xi} \subseteq^{*} D$. Indeed, we can take, for $\omega \leq \xi<\alpha$, an $f_{\xi} \in{ }^{\omega} \omega$ such that for each $n, C_{\xi}-A_{n} \subseteq f_{\xi}(n)$. Since $\mathfrak{p}=\mathfrak{c}, \mathfrak{b}=\mathfrak{c}$, hence we can choose $f$ which is an upper bound of $\left\{f_{\xi}: \omega \leq \xi<\alpha\right\}$ in $\left({ }^{\omega} \omega,<^{*}\right)$. We assume $f$ is strictly increasing. Let $D=\bigcup_{n \in \omega} A_{n} \cap f(n)$. It is not difficult to see that $D$ works. By the choice of $D$, we know that $D$ is not a member of $p$. Let $B_{\alpha}=A_{\alpha} \cap B_{\omega}-D$. Let $C_{\alpha}$ be any pseudo-intersection of $\left\{B_{\xi}: \xi \leq \alpha\right\}$. It is easy to check that $B_{\alpha}$ and $C_{\alpha}$ satisfies the requirements.

Next we will use the family $\left\{B_{\alpha}, C_{\alpha}: \alpha \in \mathfrak{c}\right\}$ to construct a topology $\tau$ on $X=$ $\omega \cup((\mathfrak{c}+1) \times\{0\})$ by assigning a neighbourhood system for each point. First of all, $\omega$ is an open discrete dense subset of $\langle X, \tau\rangle$. For $\alpha<\mathfrak{c},\left\{\left(C_{\alpha}-n\right) \cup\{\langle\alpha, 0\rangle\}: n \in \omega\right\}$ is 
a neighbourhood base for $\langle\alpha, 0\rangle$. Let $\left\{\left(B_{\alpha}-n\right) \cup\{\langle\xi, 0\rangle: \alpha \leq \xi \leq \mathfrak{c}\}: \alpha \in \mathfrak{c}, n \in \omega\right\}$ be a neighbourhood base for $\langle\mathfrak{c}, 0\rangle$. It is easy to see that $\langle X, \tau\rangle$ is a zero-dimensional Hausdorff pseudoradial space and the subspace $\omega \cup\{\langle\mathfrak{c}, 0\rangle\}$ is homeomorphic to $\omega \cup\{p\}$.

Thus we proved

Theorem 2.2 $(\mathrm{CH})$. For every point $p$ in $\omega^{*}, \omega \cup\{p\}$ can be embedded in a zerodimensional pseudoradial space.

By Theorem 3.1 in the next section, the above lemma is not always true in ZFC.

\section{In COHEN MOdEL}

We assume $\mathrm{CH}$ is true in this section.

Theorem 3.1 $(\mathrm{CH})$. If $\mathbb{P}=F n\left(\omega_{2}, 2\right)$, then in $V^{\mathbb{P}}$, for every P-point $p$, the space $\omega \cup\{p\}$ cannot be embedded in any regular pseudoradial space.

Let us just remark that the theorem remains valid if $\mathrm{CH}$ is dropped and $\kappa$ many Cohen reals are added for any regular $\kappa>\mathfrak{c}$.

Proof. Suppose

$q \Vdash$ " $\dot{p}$ is an ultrafilter on $\omega$ and $\omega \cup\{\dot{p}\}$

is embedded into a regular pseudoradial space $\langle X, \dot{\tau}\rangle "$.

We have to prove that $q \not \forall$ " $\dot{p}$ is a P-point ". We will work in $V^{\operatorname{Fn}(\lambda, 2)}$, where $\lambda$ is $M \cap \omega_{2}$ for some elementary submodel of $H_{\theta}$ for a certain regular cardinal $\theta$. We prove some preliminary facts. We will assume $\mathrm{CH}$ is true in the remainder of the section. Without loss of generality, we assume that $X$ is in the ground model and we identify $\omega \cup\{\dot{p}\}$ with its copy in $X$. We furthermore assume that $\dot{p}$ has the form $\left\{\{\pi\} \times A_{\pi}: \pi \in \operatorname{dom}(\dot{p})\right\}$, where $\pi$ is a nice name for a subset of $\omega$. Let $\dot{\mathcal{I}}$ be a nice $\mathbb{P}$-name such that

$$
\mathbf{1} \Vdash \text { “立 }=\left\{I \in[\omega]^{\omega}: I \text { converges in }\langle X, \dot{\tau}\rangle\right\} " .
$$

We also fix a sufficiently large regular cardinal $\theta$ and an elementary submodel $M$ of $H_{\theta}$ such that the following are true:

(i) $M^{\omega} \subseteq M$,

(ii) $\{\mathbb{P}, X, \dot{p}, \dot{\tau}, \dot{\mathcal{I}}\} \subset M$ and

(iii) $M$ has cardinality $\aleph_{1}$.

Let $\mathbb{P}_{M}=\mathbb{P} \cap M, X_{M}=X \cap M$ and $\lambda=M \cap \omega_{2}$. For each $\mathbb{P}$-name $\dot{Y} \in M$, if $\mathbf{1} \Vdash_{\mathbb{P}}$ " $\dot{Y} \subseteq X$ ", we can define a $\mathbb{P}_{M}$-name $\dot{Y}_{M}$ as follows. For each $x \in X_{M}$, let $A_{x}$ be a maximal antichain in $\left\{q \in \mathbb{P}_{M}: q \Vdash_{\mathbb{P}} " x \in \dot{Y} "\right\} . \mathbb{P}_{M}$ is actually $\operatorname{Fn}(\lambda, 2)$; therefore $\mathbb{P}_{M}$ is completely embedded in $\mathbb{P}$. Hence $A_{x}$ is also a maximal antichain in $\left\{q \in \mathbb{P}: q \Vdash_{\mathbb{P}}\right.$ " $\left.x \in \dot{Y} "\right\}$. Since $M^{\omega} \subseteq M$ and $\mathbb{P}$ is $c c c$, we can choose $A_{x}$ from $M$. Let $\dot{Y}_{M}$ be $\left\{\{x\} \times A_{x}: x \in X_{M}\right\}$. If, on the other hand, $\dot{\mathcal{Y}}$ is a $\mathbb{P}$-name such that $\mathbf{1} \Vdash_{\mathbb{P}}$ " $\dot{\mathcal{Y}} \subseteq \mathcal{P} X$ ", we define a $\mathbb{P}_{M}$-name $\dot{\mathcal{Y}}_{M}$ to be

$$
\left\{\left\langle\dot{Y}_{M}, \mathbf{1}\right\rangle: \dot{Y} \in M \text { and } \mathbf{1} \Vdash_{\mathbb{P}} \text { " } \dot{Y} \in \dot{\mathcal{Y}} "\right\} \text {. }
$$

Fact 3.2. Suppose $G$ is a $\mathbb{P}$-generic over $V$, and $\sigma \in M$ such that $\mathbf{1} \Vdash_{\mathbb{P}}$ " $\sigma \subseteq X$ ", then the following hold:

(i) if $\mathbf{1} \Vdash_{\mathbb{P}}$ " $\sigma \subseteq \omega$ ", then $\sigma_{M} \in M$; moreover, if $\sigma$ is a nice name, we can define $\sigma_{M}$ to be $\sigma$; 
(ii) $V[G] \models " v a l\left(\sigma_{M}, G\right)=\operatorname{val}\left(\sigma_{M}, M \cap G\right)=\operatorname{val}(\sigma, G) \cap M "$; and

(iii) if $\mathbf{1} \Vdash_{\mathbb{P}}$ " $\dot{p}$ is a P-point ", then, $\mathbf{1} \Vdash_{\mathbb{P}_{M}}$ " $\dot{p}_{M}$ is an P-point ".

Proof of Fact 3.2. (i) and (ii) are straightforward. For (iii), suppose that $\mathbf{1} \Vdash_{\mathbb{P}}$ " $\dot{p}$ is a P-point ". It is easy to see that $\mathbf{1} \Vdash_{\mathbb{P}_{M}}$ " $\dot{p}_{M}$ is an ultrafilter on $\omega "$ ". We prove that $\mathbf{1} \Vdash_{\mathbb{P}_{M}}$ " $\dot{p}_{M}$ is countably complete". Suppose $\dot{f}$ is a $\mathbb{P}_{M}$-name and $q \in \mathbb{P}_{M}$ such that $q$ forces $\dot{f}$ is a function from $\omega$ onto $\dot{p}_{M}$. For each $n \in \omega$, we can choose an antichain $B_{n}=\left\{q_{i}^{n}: i \in \omega\right\}$ which is maximal in $\left\{r \in \mathbb{P}_{M}: r \leq q\right\}$ and an associated set $\left\{\pi_{i}^{n}: i \in \omega\right\} \subseteq \operatorname{dom}\left(\dot{p}_{M}\right)$ such that $q_{i}^{n}$ forces $\dot{f}(n)=\pi_{i}^{n}$ for each $i \in \omega$. By (i), $\operatorname{dom}\left(\dot{p}_{M}\right) \subseteq M$. Therefore the $\mathbb{P}_{M}$-name $\rho=\left\{\left\langle o p\left\langle\check{n}, \pi_{i}^{n}\right\rangle, q_{i}^{n}\right\rangle: n, i \in \omega\right\}$ is in $M$, where $o p\left\langle\check{n}, \pi_{i}^{n}\right\rangle$ is a $\mathbb{P}_{M}$-name such that $\operatorname{val}\left(o p\left\langle\check{n}, \pi_{i}^{n}\right\rangle, G\right)$ is $\left\langle n, \operatorname{val}\left(\pi_{i}^{n}, G\right)\right\rangle$ (see [6]). It is obvious that $q \Vdash " \dot{f}=\rho "$.

Since $q \Vdash_{\mathbb{P}}$ " $\dot{p}$ is countably complete", we can choose a nice $\mathbb{P}$-name $\dot{Y} \in M$ such that $q \Vdash_{\mathbb{P}}$ " $\dot{Y}$ is a pseudo-intersection of $\operatorname{ran}(\rho)$ " and $\mathbf{1} \Vdash_{\mathbb{P}} " \dot{Y} \in \dot{p}$ ". By (i), we can define $\dot{Y}_{M}$ to be $\dot{Y}$. It is clear that $q \Vdash_{\mathbb{P}_{M}}$ " $\dot{Y}_{M}=\dot{Y}$ "; hence $q \Vdash_{\mathbb{P}_{M}}$ " $\operatorname{ran}(\dot{f})=\operatorname{ran}(\rho)>\dot{Y}_{M} \in \dot{p}_{M}$ ".

It is obvious that $\mathbb{P}_{M}$ is actually $\operatorname{Fn}(\lambda, 2)$. Let $\mathbb{P}_{1}$ be $\mathbb{P}_{M}$ and $\mathbb{P}_{2}$ be $\operatorname{Fn}\left(\omega_{2}-\lambda, 2\right)$, then $\mathbb{P}$ is isomorphic to $\mathbb{P}_{1} \times \mathbb{P}_{2}$. For a $\mathbb{P}$-generic filter $G$, we will also refer to $G \cap M$ as $G_{M}$ or as $G_{1}$ and $G_{2}$ will be $G \cap \mathbb{P}_{2}$ while $V_{1}$ will be $V\left[G_{1}\right]$. To further simplify the notation, we will use $Y$ to denote $\operatorname{val}(\dot{Y}, G)$ for a $\mathbb{P}$-name $\dot{Y}$. If $\dot{\mathcal{Y}}$ and $\dot{Y}$ are in $M$, we will use $Y_{M}$ and $\mathcal{Y}_{M}$ to denote $\operatorname{val}\left(\dot{Y}_{M}, G_{M}\right)$ and $\operatorname{val}\left(\dot{\mathcal{Y}}_{M}, G_{M}\right)$ respectively. Thus $p, \tau, p_{M}, \tau_{M}$, etc. are well-defined when it is clear from context which $\mathbb{P}$-generic filter $G$ is being used.

Fact 3.3. The following are true:

(i) $\mathbf{1} \Vdash_{\mathbb{P}} " \dot{p}_{M}=\dot{p} \cap V_{1}, \dot{\mathcal{I}}_{M}=\dot{I} \cap V_{1}$;

(ii) if $\rho \in M$ is a $\mathbb{P}$-name such that $\mathbf{1} \Vdash_{\mathbb{P}}$ " $\rho \subseteq \mathcal{P}(\omega)$ ", then $\mathbf{1} \Vdash_{\mathbb{P}}$ " $\rho_{M}=\rho \cap V_{1}$ "; and

(iii) $\mathbf{1} \Vdash_{\mathbb{P}_{M}}$ " $\dot{\mathcal{I}}_{M}=\left\{a \in[\omega]^{\omega}: a\right.$ converges in $\left.\left\langle X_{M}, \dot{\tau}_{M}\right\rangle\right\}$ ".

Proof of Fact 3.3. (i) is a corollary of (ii). To prove (ii), let $\rho$ satisfy the conditions of (ii) and let $G$ be a $\mathbb{P}$-generic filter over $V$. In $V[G]$, take arbitrary $a \in \operatorname{val}(\rho, G) \cap$ $V_{1}$. Let $\pi \in \operatorname{dom}(\rho)$ and $\sigma$ be a nice $\mathbb{P}_{M}$-name such that $a=\operatorname{val}(\pi, G)=\operatorname{val}(\sigma, G)$. Take a $q$ in $G$ which forces that $\pi=\sigma \in \rho$. Since $\sigma, \rho$ are in $M, q \cap M \Vdash_{\mathbb{P}}$ " $\sigma \in \rho$ ". Let $\dot{Y} \in M$ such that $\mathbf{1} \Vdash_{\mathbb{P}} " \dot{Y} \in \rho$ and $(\sigma \in \rho \rightarrow \dot{Y}=\sigma)$ "; then $\operatorname{val}\left(\dot{Y}_{M}, G\right) \in \operatorname{val}\left(\rho_{M}, G\right)$. But $q \Vdash_{\mathbb{P}} " \pi=\sigma=\dot{Y} "$. Hence $a=\operatorname{val}(\sigma, G)=$ $\operatorname{val}(\dot{Y}, G)=\operatorname{val}\left(\dot{Y}_{M}, G\right) \in \operatorname{val}\left(\rho_{M}, G\right)$. Thus we proved $\mathbf{1} \Vdash_{\mathbb{P}}$ " $\rho_{M} \supseteq \rho \cap V_{1}$ ". By the definition of $\rho_{M}$, it is obvious that

$$
\mathbf{1} \Vdash_{\mathbb{P}} " \rho_{M} \subseteq \rho \cap V_{1} " .
$$

Therefore $\mathbf{1} \Vdash_{\mathbb{P}}$ " $\rho_{M}=\rho \cap V_{1}$ ". We are left to prove (iii)

Let $H$ be a $\mathbb{P}_{M}$-generic filter over $V$; there exists a $\mathbb{P}$-generic filter $G$ over $V$ such that $H=G \cap \mathbb{P}_{M}$. We have to prove that, in $V_{1}=V\left[G_{M}\right], \mathcal{I}_{M}=\left\{a \in[\omega]^{\omega}\right.$ : a converges in $\left.\left\langle X_{M}, \tau_{M}\right\rangle\right\}$.

" $\subseteq$ ": Let $a \in \mathcal{I}_{M}$. We have to prove that a converges in $\left\langle X_{M}, \tau_{M}\right\rangle$. There exists $\dot{Y}_{M} \in \operatorname{dom}\left(\dot{\mathcal{I}}_{M}\right)$, such that $a=Y_{M}$. Since $a \subseteq \omega$, by (ii) of Fact $3.2, Y_{M}=Y$; hence $a \in M[G]$. In $V[G]$, there exists $x \in X$, such that $a$ converges to $x$ in $\langle X, \tau\rangle$. By elementarity, we can assume $x$ in $X_{M}$. Since $a \subseteq \omega \subseteq X_{M}$ and $\left\langle X_{M}, \tau_{M}\right\rangle$ is a weaker topology than $\left\langle X_{M}, \tau\left\lceil X_{M}\right\rangle, a\right.$ obviously converges to $x$ in $\left\langle X_{M}, \tau_{M}\right\rangle$. 
" $\supseteq$ ": Let $a \in V_{1} \cap[\omega]^{\omega}$ and for some $x \in X_{M}, a$ converges to $x$ in $\left\langle X_{M}, \tau_{M}\right\rangle$. We have to show that $a$ is in $\mathcal{I}_{M}$. By (i), we only have to prove that $a$ is in $\mathcal{I}$, i.e.

$$
V[G] \models \text { "a converges in }\langle X, \tau\rangle \text { ". }
$$

By the facts that $M^{\omega} \subseteq M$ and that $\mathbb{P}$ is $c c c$, any nice $\mathbb{P}_{M}$-name for a subset of $\omega$ is in $M$. In particular, $a$ is in $M[G]$. By the choice of $\theta$ and the elementarity, it is sufficient to prove that

$$
M[G] \models \text { " } a \rightarrow x \text { in }\langle X, \tau\rangle \text { " . }
$$

Let $U$ be in $\tau \cap M[G]$ containing $x$. We have to show that $a$ is almost contained in $U$. There exists $\dot{Y} \in M$ such that $U=\operatorname{val}(\dot{Y}, G)$, we can assume $\mathbf{1} \Vdash_{\mathbb{P}}$ " $\dot{Y} \in \tau$ "; hence $\dot{Y}_{M} \in \operatorname{dom}\left(\dot{\tau}_{M}\right)$. Notice that $U \cap X_{M}=U \cap M=Y_{M}$ is in $\tau_{M}$. Therefore $a$ is almost contained in $U \cap X_{M}$ therefore in $U$.

Now we are ready to finish the proof. Assume that $q$ does force $\dot{p}$ to be a P-point. We can assume that $q$ is the empty function, i.e. $q=\mathbf{1}$ ( otherwise we can work instead with $\mathbb{P}_{q}=\{r \in \mathbb{P}: r \leq q\}$ which is isomorphic to $\left.\mathbb{P}\right)$. Suppose $\theta, M$, and $\mathcal{I}$ are obtained as in the previous discussions and $G$ is a $\mathbb{P}$-generic filter over $V$. Let $a \in V[G]$ be a Cohen real over $V_{1}$ such that $a$ is in $p$. Since $\langle X, \tau\rangle$ is regular, we can take two open neighbourhoods of $p$, say $W$ and $U$, such that $\operatorname{cl}(W) \subseteq U$ and $a=U \cap \omega$. Let $b=W \cap \omega$. Given any $I$ in $\mathcal{I}_{M}$, let $x$ be in $X_{M}$ such that $I$ converges to $x$. By (i) of the Fact 3.3, $I$ also converges to $x$ in $\langle X, \tau\rangle$. If $b \cap I$ is infinite, then $x$ is in $c l(W) \subseteq U$. Therefore $I$ is almost contained in $a$. This contradicts the fact that $a$ is a Cohen real over $V_{1}$. Thus we proved that for each $I$ in $\mathcal{I}_{M}, b \cap I$ is finite. Let $\dot{\tau}, \dot{\mathcal{I}}, \dot{a}, \dot{U}, \dot{W}, \dot{b}$ and $\dot{p}$ denote both the $\mathbb{P}$-names and $\mathbb{P}_{2}$-names for the corresponding objects in $V[G]$. Choose an $r \in G_{2}$, such that $r$ forces the above fact over $V_{1}$.

Now work in $V_{1}$, let $\kappa$ be a sufficiently large regular cardinal, and let $N$ be a countable submodel of $H_{\kappa}$ such that $N$ contains $\{\dot{\tau}, \dot{\mathcal{I}}, \dot{a}, \dot{U}, \dot{W}, \dot{b}, \dot{p}, \lambda\}$. By (iii) of Fact $3.2, p_{M}$ is a P-point. We can take $A$ in $p_{M}$ such that for each $B \in N \cap p_{M}$, $A \subseteq{ }^{*} B$. Since in $V[G], A$ is in $p$ and $\langle X, \tau\rangle$ is pseudoradial, there exists $I \in \mathcal{I}$ such that $I \subset A$. Notice that $A \in M[G]$, and , according to [8], $M[G]$ is an elementary submodel of $H_{\theta}^{V[G]}$. By elementarity, we can assume $I \in M[G]$. Hence $I \in V_{1}$. Therefore, by (i) of Fact 3.3, $I \in \mathcal{I}_{M}$. By the choice of $r, r \Vdash_{\mathbb{P}_{2}}$ " $\dot{b} \cap I$ is finite", let $s \in \mathbb{P}_{2}$ and $m \in \omega$, such that $r \geq s \Vdash_{\mathbb{P}_{2}}$ " $\dot{b} \cap I \subseteq m$ ".

Claim. $s \cap N \Vdash_{\mathbb{P}_{2}}$ " $\dot{b} \cap I \subseteq m$ ". Otherwise, let $k \in I-m$, such that there is $t \leq s \cap N, t \Vdash_{\mathbb{P}_{2}}$ " $k \in \dot{b}$ ". Since $k$ and $\dot{b}$ are in $\mathrm{N}$, we can assume $t \in N$. But then $t$ and $s$ are compatible while they force contradicting statements. Therefore we can

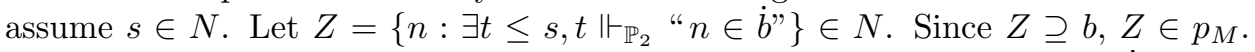
Now $I \subseteq A \subseteq * Z$, and take $k \in I \cap Z-m$ and $t \leq s$ such that $t \Vdash_{\mathbb{P}_{2}}$ " $k \in \dot{b}$ ". This contradicts the fact that $s \Vdash_{\mathbb{P}_{2}}$ " $\dot{b} \cap I \subseteq m$ ".

Remark. In a similar way, we can prove that in $V^{\mathrm{Fn}\left(\omega_{2}, 2\right)}$, for every ultrafilter $p$ on $\omega$, if $p$ is a $P$-point limit of a sequence of $P$-points, then the space $\omega \cup\{p\}$ cannot be embedded in any regular pseudoradial space.

\section{A stationary Set in $\left[\omega_{2}\right]^{\omega}$ And a special Ultrafilter}

In this section, we show that there is an ultrafilter $p$ such that $\omega \cup\{p\}$ can be embedded into a zero-dimensional Hausdorff pseudoradial space provided there is a reflecting self-indexed stationary set in $\left[\omega_{2}\right]^{\omega}$ and $\mathfrak{c}$ is $\omega_{2}$. 
$\left\{S_{\alpha}: \alpha<\omega_{2}\right\}$ is a reflecting self-indexed stationary set in $\left[\omega_{2}\right]^{\omega}$ if for any $\lambda<\omega_{2}$ with $\operatorname{cf}(\lambda)=\omega_{1},\left\{S_{\xi}: \xi<\lambda\right.$ and $\left.\sup S_{\xi}=\xi\right\}$ is stationary in $[\lambda]^{\omega}$.

The reflecting self-indexed stationary set is very similar to the notion of stationary coding set defined in [10].

Lemma 4.1. Let $V \models C H$ and $\mathbb{P}=\left\{p \subseteq \omega_{2} \times\left[\omega_{2}\right]^{\omega}: p\right.$ is a function with countable domain such that $p(\alpha) \subseteq \alpha$ for $\alpha \in \operatorname{dom} \alpha\}$. Let $\mathbb{P}$ have the inverse inclusion as its partial order. Then, in $V^{\mathbb{P}}$, there is a reflecting self-indexed stationary set in $\left[\omega_{2}\right]^{\omega}$ and $\mathrm{CH}$ is true.

It is easy to see that $\mathbb{P}$ is countably closed and $\omega_{2}$-cc. Therefore $\mathrm{CH}$ still holds in $V[P]$ and cardinals are preserved. We leave the proof to the reader.

Lemma 4.2. A reflecting self-indexed stationary set is preserved by ccc forcing.

Proof. It is well-known that after ccc forcing every cub subset of $\omega_{1}$ contains a ground model cub. The same proof gives that this also holds for cub subsets of $[\lambda]^{\omega}$ for each $\lambda<\omega_{2}$.

Remark. S. Todorcěvić informed us that the existence of a reflecting self-indexed stationary set follows from the existence of the $\rho$-function in [3]. Indeed, one can easily produce such a stationary set directly from a $\square$-sequence (see [4]).

Next we show that for the ultrafilter $p$ stated in the following Theorem, it is possible to embed $\omega \cup\{p\}$ into a zero-dimensional pseudoradial space.

Definition $4.3([2])$. Given a family $\mathcal{F}$ of subsets of some set $\mathrm{S}$, a family $\mathcal{I}$ is called a refinement of $\mathcal{F}$ if for each $F$ in $\mathcal{F}$, there is an $I$ in $\mathcal{I}$ such that $I \subseteq F$. If $\mathcal{I}$ is an almost disjoint family we say $\mathcal{I}$ is an almost disjoint refinement of $\mathcal{F}$.

Theorem 4.4 ([5]). There is an ultrafilter $p$ on $\omega$ which has an almost disjoint refinement $\mathcal{I}$ and a base $\mathcal{B}$ such that

(i) for each subset $\mathcal{I}^{\prime} \in[\mathcal{I}]^{<\mathfrak{c}}$ there is a member of $p$ which is almost disjoint with each member of $\mathcal{I}^{\prime}$,

(ii) for each $B \in \mathcal{B}$ and $I \in \mathcal{I}$ either $B \cap I$ is finite or $B$ almost contains $I$, and

(iii) each member of $\mathcal{I}$ is almost disjoint with all but finitely many members of $\mathcal{B}$.

Theorem 4.5. Suppose $p$ is the ultrafilter stated in Theorem 4.4. If $\mathfrak{c}=\omega_{2}$ and there is a reflecting self-indexed stationary set in $\left[\omega_{2}\right]^{\omega}$, then the space $\omega \cup\{p\}$ can be embedded in a zero-dimensional pseudoradial space.

Corollary 4.6. It is consistent that, for every P-point $p, \omega \cup\{p\}$ cannot be embedded into any regular pseudoradial space while there is an ultrafilter $p$ on $\omega$ such that $\omega \cup\{p\}$ is a subspace of a zero-dimensional pseudoradial space.

Proof. Let $V$ be the model obtained through Lemma 4.1. Let $Q=\operatorname{Fn}\left(\omega_{2}, 2\right)$. By Theorem 3.1, in $V^{Q}$, for every P-point $p, \omega \cup\{p\}$ cannot be embedded into any regular pseudoradial space.

On the other hand, by Lemma 4.2 , in $V^{Q}$, there is a reflecting self-indexed stationary set on $\left[\omega_{2}\right]^{\omega}$ and $\mathfrak{c}$ is $\omega_{2}$. Therefore, by Theorem 4.5 , there is an ultrafilter $p$ on $\omega$ such that $\omega \cup\{p\}$ is a subspace of a zero-dimensional pseudoradial space.

We will need the following Lemma in the proof of Theorem 4.5. 
Lemma 4.7. Suppose $R$ is a subset of space $X, x \in X$, and $\mathcal{U}$ is a local subbase at $x$, i.e, the intersections of finitely many members of $\mathcal{U}$ constitute a neighbourhood base at $x$. If $\kappa$ is any cardinal number such that the following are true,

(i) for all $\mathcal{U}^{\prime} \in[\mathcal{U}]^{<\kappa}, R \cap \bigcap \mathcal{U}^{\prime}$ is not empty;

(ii) for all $y \in R,\{U \in \mathcal{U}: y \notin U\}$ has cardinality at most $\kappa$, then there is a sequence $\left\{y_{\alpha}: \alpha<\kappa\right\}$ in $R$ convergent to $x$.

Proof. For each $y \in R$, let $\left\{U_{\alpha}^{y}: \alpha<\kappa\right\}$ enumerate, with possible repetition, the set $\{U \in \mathcal{U}: y \notin U\}$. It is straightforward to define, by the induction, a sequence $\left\{y_{\alpha}: \alpha<\kappa\right\}$ such that for all $\alpha<\kappa$

$$
y_{\alpha} \in R \cap \bigcap\left\{U_{\eta}^{y_{\xi}}: \eta<\alpha, \xi<\alpha\right\} .
$$

We show that $\left\{y_{\alpha}\right\}_{\alpha<\kappa}$ is convergent to $x$ by showing that each member of $\mathcal{U}$ contains a tail of the sequence. Suppose $U \in \mathcal{U}$. We have to show that there is an $\alpha<\kappa$, such that, for all $\beta \in \kappa, \beta>\alpha$ implies $y_{\beta} \in U$. If $\left\{y_{\alpha}: \alpha<\kappa\right\}$ is contained in $U$, we are done. Otherwise, choose any $\xi$ such that $y_{\xi} \notin U$. Take $\eta<\kappa$ such that $U=U_{\eta}^{y_{\xi}}$. Let $\alpha=\max \{\eta, \xi\}$; then, for any $\beta>\alpha$, by $(*), y_{\beta} \in U$. We are done.

The rest of the section is to prove Theorem 4.5.

Proof of Theorem 4.5. We first fix a reflecting self-indexed stationary set $\left\{S_{\alpha}: \alpha \in\right.$ $\left.\omega_{2}\right\}$ in $\left[\omega_{2}\right]^{\omega}$. Without loss of generality, we assume that, for $\alpha \in \omega_{2}$ with countable cofinality, $\alpha=\sup S_{\alpha}$. Let $p=\left\{A_{\alpha}: \alpha<\omega_{2}\right\}$ be the ultrafilter in Theorem 4.4. Let $\left\{I_{A}: A \in p\right\}$ be the almost disjoint refinement of $p$ such that $I_{A} \subseteq A$ and $\mathcal{B}$ is the base with the properties in Theorem 4.4. By induction we can define an almost disjoint family $\left\{a_{\alpha}: \alpha<\omega_{2}\right\}$ and a base $\left\{F_{\alpha}: \alpha<\omega_{2}\right\}$ of $p$ such that:

(i) $(\forall \alpha)\left(a_{\alpha} \subseteq F_{\alpha} \subseteq A_{\alpha}\right)$.

(ii) $(\forall \alpha, \beta)(\alpha<\beta)$ implies $a_{\alpha} \cap F_{\beta}$ is finite.

(iii) $(\forall \alpha)$ there are only finitely many $\beta$ such that $a_{\alpha} \cap F_{\beta}$ is infinite and if $a_{\alpha} \cap F_{\beta}$ is infinite, then $a_{\alpha} \subseteq F_{\beta}$.

(iv) $(\forall \lambda)$ if $c f(\lambda)$ is uncountable, then $\left\{F_{\alpha}: \alpha<\lambda\right\}$ is a filter base.

We construct $\left\{a_{\alpha}, F_{\alpha}: \alpha<\omega_{1} \eta\right\}$ by induction on $\eta$. At stage $\eta$, the only non trivial case is when $\eta$ is a successor ordinal. Suppose $\eta=\xi+1$. We define $a_{\omega_{1} \xi+\alpha}$ and $F_{\omega_{1} \xi+\alpha}$ for $\alpha \in \omega_{1}$ by induction on $\omega_{1}$. Let $f: \omega_{1} \rightarrow\left[\omega_{1} \xi+\omega_{1}\right]^{<\omega}$ be an onto mapping such that $f(\alpha) \subseteq \omega_{1} \xi+\alpha$ for each $\alpha \in \omega_{1}$. At stage $\alpha$, take a $B$ in $\mathcal{B}$ such that $B$ is almost disjoint with $a_{\zeta}$ for each $\zeta<\omega_{1} \xi+\alpha$. Now simply define $F_{\omega_{1} \xi+\alpha}$ to be $A_{\omega_{1} \xi+\alpha} \cap B \cap \bigcap\left\{F_{\zeta}: \zeta \in f(\alpha)\right\}$ and let $a_{\omega_{1} \xi+\alpha}$ be $I_{F_{\omega_{1} \xi+\alpha}}$.

Let $\mathcal{H}=\left\{b \subseteq \omega:\left(\forall \alpha \in \omega_{2}\right)\left(a_{\alpha} \subseteq^{*} b\right)\right\}$. Let $\mathcal{A}$ be the subalgebra of $P(\omega)$ generated by $[\omega]^{<\omega} \cup\left\{a_{\alpha}, F_{\alpha}: \alpha \in \omega_{2}\right\} \cup \mathcal{H}$.

We will define a subspace $X$ of the Stone space $\operatorname{St}(\mathcal{A})$ such that $X$ contains a copy of $\omega \cup\{p\}$ and $X$ is pseudoradial. $X$ will be of the form $\omega \cup\left\{x_{\alpha}: \alpha \in \Gamma\right\}$, where $\Gamma$ is a subset of $\omega_{2}$ and each $x_{\alpha}$ is an ultrafilter of $\mathcal{A}$. We specify the set $\Gamma$ and $x_{\alpha}$ for $\alpha \in \Gamma$ as follows.

For convenience, we define a subset $x_{\alpha}^{+}$of $\mathcal{F}$ for each $\alpha \in \omega_{2}$. For successor ordinal $\alpha+1, x_{\alpha+1}^{+}$is the finite of $F_{\beta}$ 's which contains $a_{\alpha}$. For limit $\alpha$ with $\operatorname{cf}(\alpha)=\omega, x_{\alpha}^{+}$ is $\left\{F_{\alpha}: \alpha \in S_{\alpha}\right\}$, where $S_{\alpha}$ is from the reflecting self-indexed stationary set. For limit $\lambda$ with $\operatorname{cf}(\lambda)=\omega_{1}, x_{\lambda}^{+}$is $\left\{F_{\alpha}: \alpha<\lambda\right\}$. 
First of all, $\Gamma$ contains all the successor ordinals and the ordinals with uncountable cofinality.

For each $\alpha$, since no member of $\mathcal{A}$ splits $a_{\alpha}$, the cofinite subsets of $a_{\alpha}$ generate an ultrafilter on $\mathcal{A}$. Let $x_{\alpha+1}$ be this ultrafilter. The point $x_{\omega_{2}}$ will simply be the ultrafilter $p \cap \mathcal{A}$. Before we handle other ordinals, we prove the following.

Fact 4.8. If $\lambda<\omega_{2}$ has cofinality $\omega_{1}$, then $x_{\lambda}^{+} \cup\left\{\omega-F_{\beta}: F_{\beta} \notin x_{\lambda}^{+}\right\} \cup \mathcal{H}$ is actually $\left\{F_{\alpha}, \omega \backslash F_{\beta}: \alpha<\lambda\right.$ and $\left.\beta \geq \lambda\right\} \cup \mathcal{H}$ and generates an ultrafilter $x_{\lambda}$ on $\mathcal{A}$. If $\alpha<\omega_{2}$ has cofinality $\omega$ and $x_{\alpha}^{+} \cup\left\{\omega-F_{\beta}: F_{\beta} \notin x_{\alpha}^{+}\right\} \cup \mathcal{H}$ has the finite intersection property, then it generates an ultrafilter $x_{\alpha}$ on $\mathcal{A}$.

Proof of Fact 4.8. If $\lambda<\omega_{2}$ has cofinality $\omega_{1}$, then $\left\{F_{\alpha}: \alpha<\lambda\right\}$ is a filter base and it is easy to see that $\left\{F_{\alpha}, \omega \backslash F_{\beta}: \alpha<\lambda\right.$ and $\left.\beta \geq \lambda\right\} \cup \mathcal{H}$ has the finite intersection property. To prove that it generates an ultrafilter, it is sufficient to show that for each $\beta \in \omega_{2}, \omega \backslash a_{\beta}$ contains some member of the family. Indeed, if $\beta<\lambda$, then $F_{\beta+1}$ is almost disjoint with $a_{\beta}$; hence $F_{\beta+1} \subseteq^{*} \omega \backslash a_{\beta}$. If $\beta \geq \lambda$, then $\omega \backslash F_{\beta}$ is almost contained in $\omega \backslash a_{\beta}$. The proof for the case when $\alpha$ has countable cofinality is virtually the same.

We resume the proof of the theorem. For $\lambda$ with cofinality $\omega_{1}$, let $\lambda$ be in $\Gamma$ and $x_{\lambda}$ be the ultrafilter defined in Fact 4.8. For an ordinal $\alpha$ with $\operatorname{cf}(\alpha)=\omega, \alpha \in \Gamma$ if $x_{\alpha}^{+} \cup\left\{\omega-F_{\beta}: F_{\beta} \notin x_{\alpha}^{+}\right\} \cup \mathcal{H}$ generate a filter; we let $x_{\alpha}$ be the ultrafilter defined in Fact 4.8

By above discussion $\Gamma$ is well defined and, for each $\alpha \in \Gamma, x_{\alpha}$ is a point in the Stone space $\operatorname{St}(\mathcal{A})$. The following claim is trivial.

Claim 4.1. $\omega \cup\left\{x_{\omega_{2}}\right\}$ is homomorphic to $\omega \cup\{p\}$.

For simplicity we will abuse the notation of Stone duality. If $F \subset \omega$ is a member of $\mathcal{A}$, we also let $F$ denote the set of points of $X$ which as an ultrafilter on $\mathcal{A}$ contain $F$ as a member. Of course, $F$ is clopen; hence, to say $x_{\alpha} \in F$ is equivalent to saying $F \in x_{\alpha}$.

Let $X_{0}$ be all $x_{\alpha}$ with $\alpha$ a successor and $X_{1}$ be all $x_{\alpha}$ with $\operatorname{cf}(\alpha)=\omega$. Recall that $x_{\alpha+1}$ corresponds to $a_{\alpha}$. Let $X_{2}$ be all $x_{\alpha}$ such that $\alpha$ has uncountable cofinality.

Fact 4.9. The sets $X_{1} \cup X_{2}$ and $X_{2}$ are closed in $X . X_{2}$ is homomorphic to a subspace of $\omega_{2}$, namely $\left\{\alpha \in \omega_{2}: \operatorname{cf}(\alpha)\right.$ is uncountable $\}$ and so $X_{2}$ is radial.

Proof of Fact 4.9. For the first statement of the claim, the only case that requires proof is that a point $x_{\alpha}$ of $X_{1}$ is not in the closure of $X_{2}$. If $\alpha$ is countable, then $\omega-F_{\alpha+1}$ is disjoint from $X_{2}$. On the other hand, if $\alpha$ is uncountable, then we can find $\gamma<\beta<\alpha$, so that $F_{\beta} \in x_{\alpha}^{+}$and $F_{\gamma} \notin x_{\alpha}^{+}$. Note that $F_{\beta} \backslash F_{\gamma}$ is not a member of $x_{\lambda}$ for any $\lambda$ with uncountable cofinality. For the second statement, it is sufficient to see that, for any $\lambda<\omega_{2}$ with uncountably cofinality, the typical neighhourhood of $x_{\lambda}$ in the subspace $X_{2}$ is $\left(F_{\alpha} \backslash F_{\lambda}\right) \cap X_{2}=\left\{x_{\xi} \in X_{2}: \alpha<\xi \leq \lambda\right\}$ for some $\alpha<\lambda$. Similarly, a typical neighbourhood of $x_{\omega_{2}}$ is $\left\{x_{\xi} \in X_{2}: \alpha<\xi \leq \omega_{2}\right\}$.

Claim 4.2 will finish the proof of Theorem 4.5 .

Claim 4.2. $X$ is a zero-dimensional pseudoradial space.

Proof of Claim 4.2. Since $X$ is a subspace of a $\operatorname{St}(\mathcal{A}), X$ is zero-dimensional. Suppose $X$ is not pseudoradial. Let $R$ be a radially closed subset of $X$ which is not 
closed. Let $x_{\alpha} \in \operatorname{cl} R \backslash R$ and $\alpha$ is the minimal such index. Obviously $x_{\alpha} \in X_{1} \cup X_{2}$ because other points have countable neighbourhood bases. We will prove $x_{\alpha} \in R$ to produce a contradiction. We will need the following fact.

Fact 4.10. $x_{\alpha} \in \operatorname{cl}(R \backslash \omega)$.

Proof of Fact 4.10. Suppose $x_{\alpha} \notin \operatorname{cl}(R \backslash \omega)$. Then $x_{\alpha} \in \operatorname{cl}(R \cap \omega) \backslash \operatorname{cl}(R \backslash \omega)$. Let $U$ be a clopen neighbourhood of $x_{\alpha}$ such that $U \cap(R \backslash \omega)=\emptyset$. Hence $b=R \cap U$ is a subset of $\omega$. Since $R$ is radially closed and $U$ is closed, $b$ is also radially closed. For any $\beta \in \omega_{2}$, if $a_{\beta} \cap b$ is infinite, then $x_{\beta+1}$ would be in $b$ contradicting that $b \subseteq \omega$. Hence, $b$ is almost disjoint with each $a_{\beta}$, i.e, $\omega \backslash b$ is in $\mathcal{H}$. Thus $\omega \backslash b$ are in the ultrafilter $x_{\alpha}$, which contradict that $x_{\alpha}$ is in the closure of $b$.

Since $R \backslash \omega$ is also radially closed in $X$, we can now assume $R \cap \omega=\emptyset$. By Fact 4.9, $X_{2}$ is radial and closed so we can assume that $R \cap X_{2}=\emptyset$ by again restricting to a neighbourhood of $x_{\alpha}$. For the rest of the proof we assume that $R \subseteq X_{0} \cup X_{1}$. Recall that $x_{\alpha} \in X_{1} \cup X_{2}$.

We claim that $\alpha \neq \omega_{2}$. Indeed fix any $M \prec H\left(\omega_{3}\right)$ such that $|M|=\omega_{1}$ and $M$ contains all relevant sets such as $R$ and $X$, and so that the cofinality of $\delta=M \cap \omega_{2}$ is uncountable. We show that $x_{\delta}$ is in the closure of $R$ which will contradict that $R$ is assumed to be disjoint from $X_{2}$ and that $\alpha$ is the minimal index of a limit point which is not in $R$. A typical neighbourhood of $x_{\delta}$ has the form $H \cap \bigcap\left\{F_{\xi}: \xi \in f\right\} \backslash \bigcup\left\{F_{\gamma}: \gamma \in g\right\}$ where $f \in[\delta]^{<\omega}, g \in\left[\omega_{2} \backslash \delta\right]^{<\omega}$ and $H \in \mathcal{H}$. However, $\bigcap\left\{F_{\xi}: \xi \in f\right\}$ is a neighbourhood of $x_{\omega_{2}}$ and is a member of $M$. Choose any $\beta \in M$ such that $x_{\beta} \in R \cap \bigcap\left\{F_{\xi}: \xi \in f\right\}$. Clearly $x_{\beta} \notin F_{\gamma}$ for all $\gamma>\delta$; hence $x_{\beta}$ is in the above neighbourhood of $x_{\delta}$.

Next we apply Lemma 4.7 to prove that there exists a sequence in $R$ convergent to $x_{\alpha}$ and thus finish the proof.

Case 1. $\operatorname{cf}(\alpha)=\omega$.

To apply Lemma 4.7 , let $\kappa$ be $\omega, \mathcal{U}$ be $x_{\alpha}^{+} \cup\left\{\omega \backslash F_{\beta}: x_{\beta} \notin x_{\alpha}^{+}\right\} \cup \mathcal{H}$, and $x$ be $x_{\alpha}$. $\mathcal{U}$ is a local subbase of $x$. We show $x, R$ and $\mathcal{U}$ satisfy the conditions in Lemma 4.7. Since $x \in \operatorname{cl}(R)$, the condition $(i)$ is trivial. To verify the condition (ii), let $x_{\xi} \in R$. Then $\operatorname{cf}(\xi) \leq \omega$. It is easy to see that

$$
\left\{U \in \mathcal{U}: x_{\xi} \notin U\right\} \subseteq x_{\alpha}^{+} \cup\left\{\omega \backslash F: F \in x_{\xi}^{+}\right\} .
$$

But both $x_{\alpha}^{+}$and $x_{\xi}^{+}$are countable. Therefore $\left\{U \in \mathcal{U}: x_{\xi} \notin U\right\}$ is countable.

Case 2. $c f(\alpha)=\omega_{1}$.

We let $\kappa$ be $\omega_{1}$, and $\mathcal{U}$ be $x_{\alpha}^{+} \cup\left\{\omega \backslash F_{\beta}: x_{\beta} \notin x_{\alpha}^{+}\right\} \cup \mathcal{H}$, and $x$ be $x_{\alpha}$. Again $\mathcal{U}$ is a local subbase at $x$. The second condition of Lemma 4.7 is verified as in the Case 1. We are left to show that for any $\mathcal{U}^{\prime} \in[\mathcal{U}]^{\omega}, R \cap \cap \mathcal{U}^{\prime} \neq \emptyset$. Since for each $H$ in $\mathcal{H}$, $R \subseteq X \backslash \omega \subseteq H$, we can assume $\mathcal{U}^{\prime}=\left\{F_{\alpha_{i}}, \omega \backslash F_{\beta_{i}}: i \in \omega\right\}$. We take a countable elementary submodel $M$ of $H\left(\omega_{3}\right)$, such that $\left\{X, R, \mathcal{U}^{\prime}, \alpha\right\} \subseteq M$ and $M \cap \alpha=S_{\gamma}$ and $\gamma=\sup (M \cap \alpha)$, where $S_{\gamma}$ is from the reflecting self-indexed stationary set. Since $S_{\gamma}=M \cap \alpha$ and $x_{\gamma}^{+}$is a filter base, $x_{\gamma}^{+} \cup\left\{\omega \backslash F_{\xi}: F_{\xi} \notin x_{\gamma}^{+}\right\} \cup \mathcal{H}$ has the finite intersection property. Therefore it generates an ultrafilter on $\mathcal{A}$. Therefore $\gamma \in \Gamma$, i.e., $x_{\gamma}$ is a point of our $X$. Next we show that $x_{\gamma} \in R \cap \cap \mathcal{U}^{\prime}$.

For $i \in \omega$, since, $\mathcal{U}^{\prime} \in M, \alpha_{i} \in M \cap \alpha=S_{\gamma}$. Thus, $F_{\alpha_{i}} \in x_{\gamma}^{+}$while $F_{\beta_{i}} \notin x_{\gamma}^{+}$ because $\beta_{i} \geq \alpha>\gamma$. This proves that $x_{\gamma} \in \bigcap \mathcal{U}^{\prime}$. 
To prove $x_{\gamma} \in R$, it is sufficient to prove that $x_{\gamma} \in \operatorname{cl}(R)$ because $\alpha$ is the minimal index such that $x_{\alpha} \in \operatorname{cl}(R) \backslash R$. Take a neighbourhood $W$ of $x_{\gamma}$; we have to show $R \cap W \neq \emptyset$. We can assume $W=\bigcap \mathcal{F} \backslash \cup \mathcal{G}$, where $\mathcal{F}$ and $\mathcal{G}$ are finite subsets of $x_{\gamma}^{+}$and $\mathcal{B} \backslash x_{\gamma}^{+}$respectively. Obviously $\mathcal{F}$ and $\mathcal{G} \cap M$ are members of $M$. If $F_{\xi} \in \mathcal{G} \cap M$, then by the definition of $x_{\gamma}^{+}$and the fact $S_{\gamma}=M \cap \alpha, \xi \geq \alpha$. Therefore, $(R \cap \cap \mathcal{F}) \backslash \bigcup(\mathcal{G} \cap M)$ is a neighbourhood of $x_{\alpha}$ and it is in $M$. Take a $x_{\xi} \in M \cap R \cap \cap \mathcal{F} \backslash \bigcup(\mathcal{G} \cap M)$. Since $x_{\xi}^{+}$is a countable member of $M, x_{\xi}^{+} \subseteq M$; hence $\mathcal{G} \backslash M$ is disjoint with $x_{\xi}^{+}$. Therefore $x_{\xi} \notin \bigcup(\mathcal{G} \backslash M)$ and $x_{\xi} \in R \cap \cap \mathcal{F} \backslash \bigcup \mathcal{G}=R \cap W$. We are done.

We finish with the following question.

Question 4.11. Does $M A$ or $\mathfrak{p}=\mathfrak{c}$ imply that, for each ultrafilter $p$ on $\omega, \omega \cup\{p\}$ is a subspace of a regular pseudo-radial space?

By Lemma 2.1, the answer is yes for the non P-point $p$.

\section{REFERENCES}

[1] A.V. Arhangel'skiǔ, R. Isler, and G.Tironi. On pseudo-radial spaces. Comment. Math. Univ. Carolinae, 27(1):137-154, 1986.

[2] Bohuslav Balcar and Petr Simon. Disjoint refinement. In J.K. Monk and R. Bonnet, editors, Handbook of Boolean Algebra, chapter 9. Elsevier Science Publishers, B.V., 1989. CMP 21:10

[3] Mohamed Bekkali. Topics in Set Theory. Springer-Verlag, 1991. MR 92m:03070

[4] Alan Dow. The completion of $\mathcal{P}(\omega) /$ Fin is not equal to the regular open algebra of $\beta \mathbb{R} \backslash \mathbb{R}$. to appear.

[5] Alan Dow and Jinyuan Zhou. Two real ultrafilters on $\omega$. on Topology and its Applications. to appear

[6] Kenneth Kunen. Set Theory. Elsevier Science Publishers, B.V., 1980. MR 82f:03001

[7] Peter J. Nyikos. Convergence in topology. In M. Husěk and J. van Mill, editors, Recent Progress in General Topology, chapter 17. Elsevier Science Publishers B.V., 1992. CMP 93:15

[8] Saharon Shelah. Proper Forcing. Springer-Verlag, 1982. MR 84h:03002

[9] Jinyuan Zhou. On subspaces of pseudo-radial spaces. Comment. Math. Univ. Carolinae, 34(3):583-586, 1994. MR 94h:54003

[10] William S. Zwicker. $P_{\kappa} \lambda$ combinatorics I: Stationary coding sets rationalize the club filter. In Donald A. Martin James E. Baumgartner and Saharon Shelah, editors, Axiomatic Set Theory, pages 243-259. American Mathematical Society, 1984. MR 86e:03046

Department of Mathematics, York University, 4700 Keele Street, North York, OnTARIO, CANADA M3J 1P3

E-mail address: Alan.Dow@mathstat.yorku.ca

E-mail address: jzhou@spicer.com 